\title{
Article \\ Enhancement of Terahertz Radiation by Surface Plasmons Based on CdTe Thin Films
}

\author{
Huiyan Kong ${ }^{1} \mathbb{D}$, Luyi Huang ${ }^{2}$, Min Li $^{1}{ }^{\text {, Ling Zhang }}{ }^{1, *}$ and Heping Zeng ${ }^{3} \mathbb{D}$ \\ 1 School of Optical-Electrical and Computer Engineering, University of Shanghai for Science and Technology, \\ Shanghai 200093, China; 192380339@st.usst.edu.cn (H.K.); minli_1220@163.com (M.L.) \\ 2 CAS Key Laboratory of Nanophotonic Materials and Devices \& Key Laboratory of Nanodevices and \\ Applications, i-Lab, Suzhou Institute of Nano-Tech and Nano-Bionics (SINANO), \\ Chinese Academy of Sciences, Suzhou 215123, China; luhuang2021@sinano.ac.cn \\ 3 State Key Laboratory of Precision Spectroscopy, East China Normal University, Shanghai 200062, China; \\ hpzeng@phy.ecnu.edu.cn \\ * Correspondence: lzhang@usst.edu.cn; Tel.: +86-183-0192-5823
}

Citation: Kong, H.; Huang, L.; Li, M.; Zhang, L.; Zeng, H. Enhancement of Terahertz Radiation by Surface Plasmons Based on CdTe Thin Films. Nanomaterials 2022, 12, 290. https:// doi.org/10.3390/nano12020290

Academic Editor: Daniela Iacopino

Received: 11 December 2021

Accepted: 12 January 2022

Published: 17 January 2022

Publisher's Note: MDPI stays neutral with regard to jurisdictional claims in published maps and institutional affiliations.

Copyright: (C) 2022 by the authors. Licensee MDPI, Basel, Switzerland. This article is an open access article distributed under the terms and conditions of the Creative Commons Attribution (CC BY) license (https:// creativecommons.org/licenses/by/ $4.0 /)$.

\begin{abstract}
Terahertz (THz) time-domain spectroscopy (TDS) is a powerful tool used to characterize the surface/interface of materials, and semiconductor/metal interfaces can generate $\mathrm{THz}$ emission through ultrafast optical excitation, which can be further improved through the optical excitation of surface plasmons. Here, we assembled cadmium telluride (CdTe) on an $\mathrm{AuAg}$ alloy $\left(\mathrm{Au}_{25} \mathrm{Ag}_{75}\right.$, wt.\%) substrate and obtained five times stronger $\mathrm{THz}$ emission compared with silicon substrate, and found that the enhancement can be tuned by controlling the thickness of the semiconductor materials and plasmonic metal substrates. We believe that our results not only promote the development of $\mathrm{THz}$ emission enhancement, but also provide a straightforward way of producing small, thin, and more efficient terahertz photonic devices.
\end{abstract}

Keywords: THz emission time-domain spectroscopy; cadmium telluride; metals; THz enhancement; surface plasmons

\section{Introduction}

Terahertz (THz) technology possesses the unique properties of low energy, a lack of damage, and the unique fingerprint characteristics of non-polar molecules. It has blossomed rapidly through the development of ultrafast optical technologies and hybrid materials, and has been applied in various fields [1-6], such as biological imaging, medical diagnosis, customs security, and high-speed communication. Therefore, there is an increasing demand for high-efficiency THz emission sources and high-sensitivity THz wave detectors. Semiconductor materials, such as layered centrosymmetric $2 \mathrm{H}-\mathrm{MoS}_{2}$ (TMDs), InAs, graphene, and Ge crystals, feature high carrier mobility, wide band range, high current on/off and other photoelectric properties, and have been used as sources of electromagnetic radiation in the $\mathrm{THz}$ range [7-11]. Additionally, THz emission strength and efficiency can be further improved by constructing semiconductor/metal Schottky interfaces. Ramakrishnan et al. prepared $\mathrm{a} \mathrm{Cu}_{2} \mathrm{O} / \mathrm{Au}$ structure to increase $\mathrm{THz}$ emission and pointed out that excitation of plasmons at the interface between semiconductor and metal increased light concentration and absorption [12]. Bahk et al. reported that an emitted THz wave of graphene deposited on a gold substrate was significantly enhanced by localized surface plasmons, and they doped out a terahertz emission mechanism that related to surface-plasmon-enhanced optical rectification [13]. Huang et al. demonstrated that a surface plasmon from nano-porous gold could promote photocurrent density in cadmium telluride (CdTe) to further enhance THz emission [14].

In this study, we constructed a semiconductor/metal by depositing thin CdTe films on gold and $\mathrm{Au}_{25} \mathrm{Ag}_{75}$ alloy substrates with rough surfaces, and achieved nearly five times the 
enhancement of terahertz radiation compared with $\mathrm{CdTe}$ on silicon. We argue that both the thickness of CdTe and the components of metal substrates affect THz emission efficiency.

\section{Materials and Methods}

Thin CdTe [15] films were deposited on silicon (Si, 100), gold (Au), and AuAg alloy $\left(\mathrm{Au}_{25} \mathrm{Ag}_{75}\right.$, wt.\%) substrates through thermal evaporation deposition at room temperature [16]. Au and AuAg substrates were obtained by using a magnetron sputtering instrument on silicon wafers with a chromium layer for buffering at room temperature. All the metal targets were provided by Zhongnuo New Materials (Beijing, China) Technology Company. CdTe powder (Leshan Kai Yada Photoelectric Technology Co. Ltd, Leshan, Sichuan, China; $99.999 \%$ purity) was used as the evaporator source evaporated from a tungsten-wire-heated quartz boat. The thickness of the thin CdTe films was dominated by a quartz crystal monitor (FTM106) with a deposition rate of about $2 \AA / \mathrm{s}$. During the process of evaporation, the vacuum pressure of the chamber was lower than $10^{-4} \mathrm{~Pa}$, and the substrates kept rotating at $10 \mathrm{rad} / \mathrm{s}$ to improve the uniformity of the CdTe films. CdTe films with thicknesses of $\sim 5, \sim 10$, and $\sim 15 \mathrm{~nm}$ were obtained by controlling evaporating time [14]. The progress of preparing samples is shown in Figure 1a.

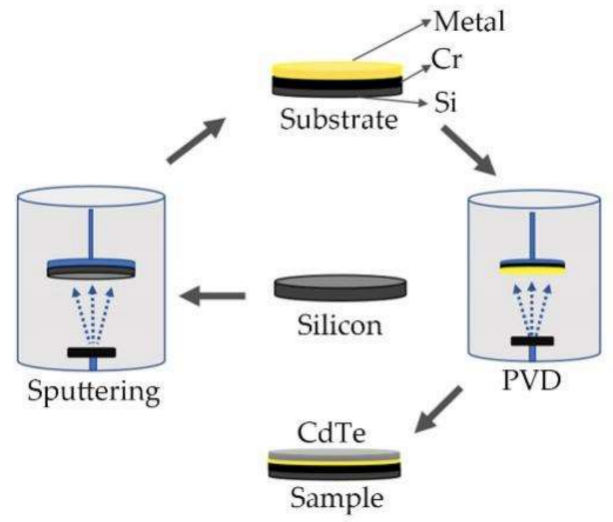

(a)

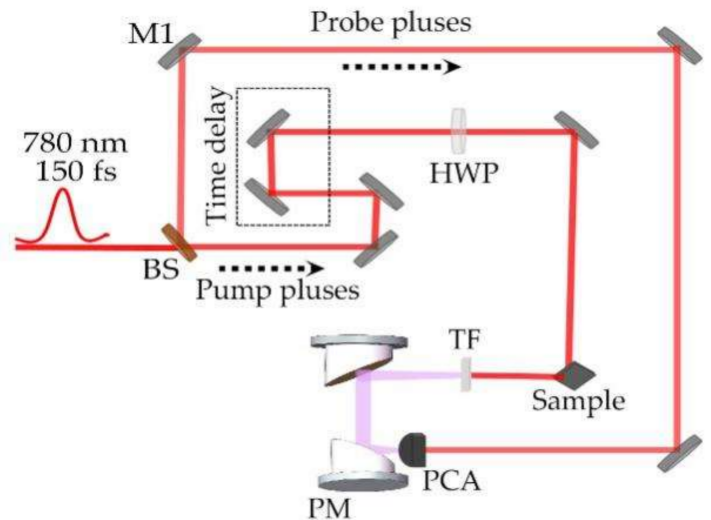

(b)

Figure 1. (a) Process of sample preparation. PVD: Physical vapor deposition; (b) schematic of a THz emission spectroscopy system. BS: beam splitter; M1: mirror; PM: paraboloidal mirror; HWP: half-wave plate; PCA: Photoconductive antenna; TF: Teflon wafer.

Home-made THz time-domain spectroscopy (TDS) was used for sample measurement and a schematic of the system is shown in Figure 1b. This system used an ultrafast laser with $780 \mathrm{~nm}, 150$ femtosecond pulse duration and $80 \mathrm{MHz}$ repetition rate. An average pump power of $80 \mathrm{~mW}$ was used for irradiating samples. During the measurement, laser pulse first traveled through a beam splitter and split into two parts that were used as the pump beam with the majority of the pulse energy and the probe beam with weak energy; subsequently, the pump beam passed through a time delay system that consisted of a pair of mirrors and an optical precision moving platform to change the relative time delay between the pump beam and the probe beam. The pump beam was focalized into a spot area of $\sim 50 \mu \mathrm{m}^{2}$ and then irradiated the surface of samples in a $45^{\circ}$ angle direction. The remaining pump beam was prevented by a Teflon wafer, and the generated $\mathrm{THz}$ pulse was focused by a pair of parabolic mirrors. Finally, the $\mathrm{THz}$ electromagnetic wave was collected onto a photoconductive antenna (PCA, Jena, Germany; BATOP GmbH), which was excited by the probe beam, and was transmitted into the phase-locked amplifier (Stanford SR830, Stanford, SRS) and shown by the domain-time waveforms of $\mathrm{THz}$ radiation.

\section{Results and Discussions}

The surface morphologies of the Au and AuAg films without and with CdTe covering were characterized with a scanning electronic microscope (SEM, Hitachi, Tokyo, Japan) and shown in Figure 2a. It was found that the surfaces of $\mathrm{Au}$ and $\mathrm{AuAg}$ film were uniform, 
with similar roughness, and the thicknesses of the $\mathrm{Au}$ and $\mathrm{AuAg}$ layers were around 170 and $180 \mathrm{~nm}$, respectively. After CdTe evaporation, nanoparticles were observed on the surface of the substrates. The Raman spectra of CdTe on Au and AuAg substrates were of the same shape, but the intensity collected from CdTe@Au was less than half of that from CdTe@AuAg. The characteristic Raman peak at $95 \mathrm{~cm}^{-1}$ indicated the low-intensity mode of CdTe [17] and the peak of $164 \mathrm{~cm}^{-1}$ corresponded to the LO mode from CdTe vibration [18-20]. The $141 \mathrm{~cm}^{-1}$ peak resulted in a combination of TO and $\mathrm{E}$ phonon modes of $\mathrm{Cd}$, and the peak at $122 \mathrm{~cm}^{-1}$ was related to the $\mathrm{A}_{1}$ mode of Te in CdTe. Therefore, the main component of the deposited film was CdTe.
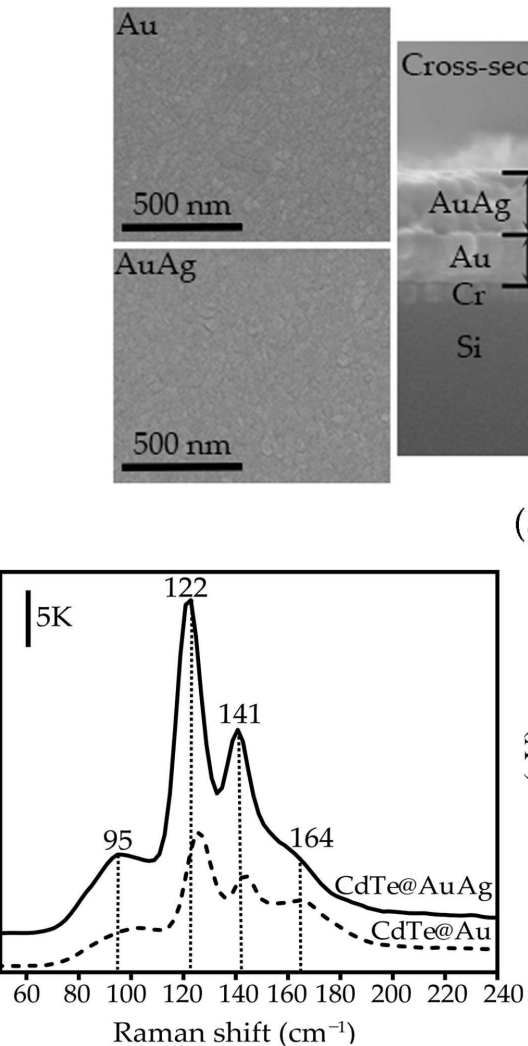

(b)

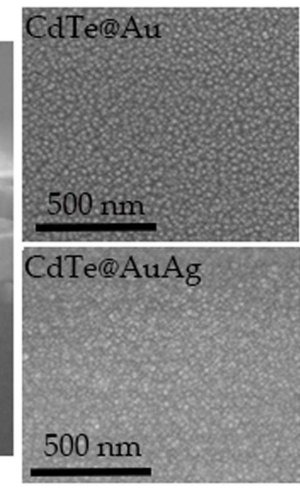

(a)

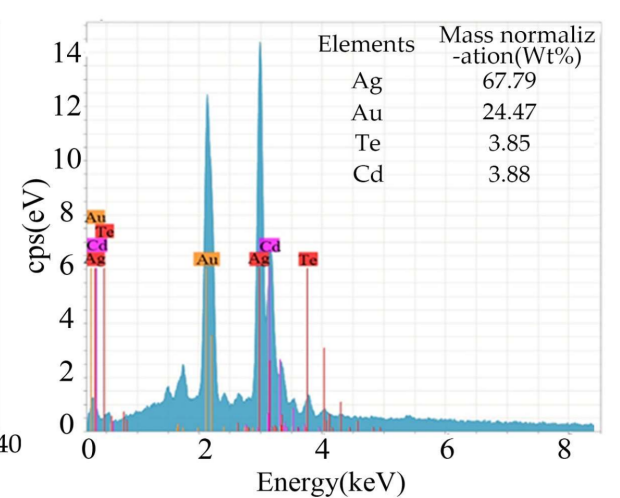

(c)

Figure 2. (a) SEM images of Au and AuAg films without and with CdTe covering, and the crosssection of the metal substrate; (b) Raman spectra of CdTe on Au and AuAg (the excitation wavelength of the laser is $532 \mathrm{~nm}$ with power set at $1 \mathrm{~mW}$ ); (c) EDS spectrum of CdTe@AuAg and the element percentages of $\mathrm{Cd}$ and $\mathrm{Te}$ are shown in the top-right corner.

Energy-dispersive X-ray spectroscopy (EDS, Tokyo, Hitachi) was adopted to verify the composition of the sample [21]. As shown in Figure 2c, the energy levels of the Cd and Te elements were detected, and the atomic percentages of $\mathrm{Cd}$ and Te were $\sim 3.88$ and $\sim 3.85 \%$, respectively, which further confirmed that the main component of the evaporated films is CdTe.

When the laser light beam impinged on the CdTe films, the optical absorption led to the photo-excited electron-hole pairs transiting from the valence band to the conduction band to serve as the photocarrier. This carrier, accelerated by intrinsic electric fields, generates $\mathrm{THz}$ wave emission [22]. It can be seen from Figure 3 a that the $\mathrm{THz}$ radiation signals of thin $\mathrm{CdTe}$ film on different substrates were different. By comparing the THz signals of CdTe on the $\mathrm{Si}, \mathrm{Au}$, and $\mathrm{AuAg}$ alloy substrates, we found that the $\mathrm{THz}$ radiation amplitudes of $\mathrm{CdTe}$ on the AuAg and Au films were enhanced by $\sim 5$ times and $\sim 2.5$ times compared to that on the $\mathrm{Si}$. For an analogous comparison in the frequency domain, the corresponding Fourier-transformed spectrum (shown in Figure 3a, inset) featured a bandwidth of about 
1.6 $\mathrm{THz}$. The results indicated that noble metal can enhance the $\mathrm{THz}$ radiation of $\mathrm{CdTe}$ because the electrons in the metal were excited by the laser beam, and then accumulated to form surface plasmon polaritons (SPPs) [23]. A strong localized surface electromagnetic field was generated, boosting the absorption of laser light. Due to the influence of the localized electromagnetic field, the internal electron mobility and the charge density in CdTe were adjusted again to further increase the surface depletion electric field, similar to the photo-Dember effect. With photoexcited carriers to the conduction band, both the localized electromagnetic field at the CdTe and metal interface and the intrinsic surface electric field from CdTe drove the charge transport to improve the efficiency of $\mathrm{THz}$ emission on the surface/interface of the material [12,24]. Meanwhile, the THz amplitude signal of CdTe@AuAg was 2 times stronger than that of CdTe@Au. It was shown that binary alloy exhibits greater enhancement than pure metal. Compared with $\mathrm{Au}$, Ag features higher electrical and thermal conductivities at room temperature and exhibits high-density photo-generated electrons. However, $\mathrm{Au}$ is more stable and can integrate with $\mathrm{CdTe}$ better due to its lower Schottky barrier $[21,25,26]$, which results in higher carrier transmission and power conversion efficiency. Thus, AuAg alloy, which combines the best of both metals, is a good candidate for the plasmonic substrate.

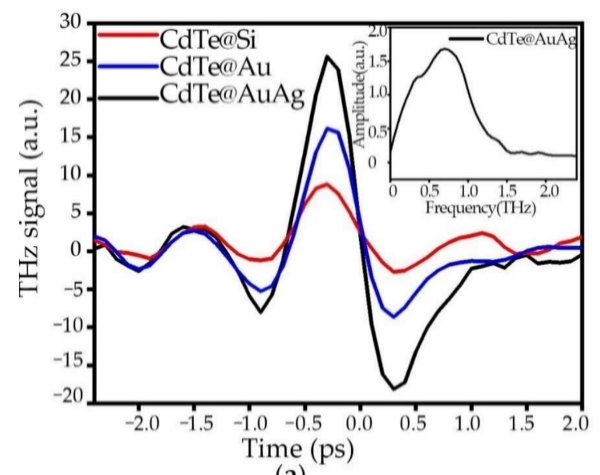

(a)

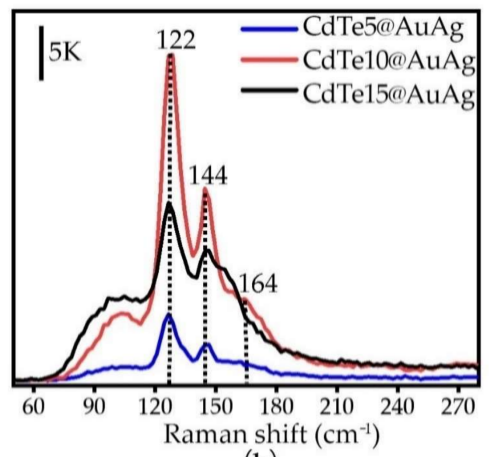

(b)

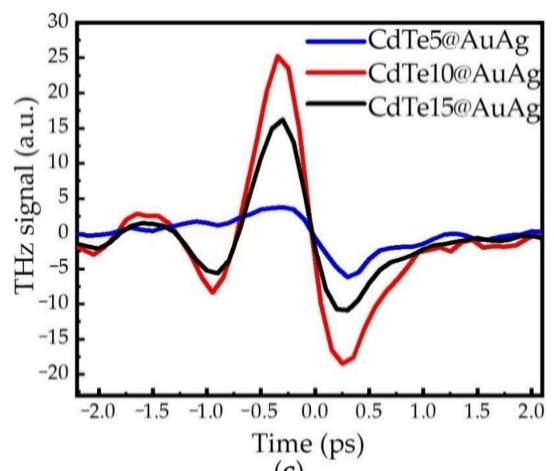

(c)

Figure 3. (a) THz emission waveforms of $\sim 10 \mathrm{~nm}$ CdTe film on the $\mathrm{Si}$, $\mathrm{Au}$, and AuAg substrates in time-domain, and the inset is the corresponding frequency spectrum of CdTe@AuAg film; (b) Raman spectra of CdTe5@AuAg (the thickness of CdTe is $5 \mathrm{~nm}$ ), CdTe10@AuAg (the thickness of CdTe is $10 \mathrm{~nm}$ ), CdTe15@AuAg (the thickness of CdTe is $15 \mathrm{~nm}$ ); (c) THz emission waveforms from CdTe5@AuAg,CdTe10@AuAg and CdTe15@AuAg films.

The Raman spectra (RAMANtouch, Nanophoton, Osaka, Japan) of the CdTe films on AuAg with thicknesses of 5 nm (CdTe5@AuAg), 10 nm (CdTe10@AuAg), and 15 nm (CdTe15@AuAg) were observed and shown in Figure 3b. By comparing the relative intensity of each peak, it can be seen that the intensity of the 122 and $144 \mathrm{~cm}^{-1}$ Raman peaks from CdTe10@AuAg was significantly enhanced, and was about five times larger than that from CdTe5@AuAg and 1.5 times stronger than that from CdTe15@AuAg. The corresponding $\mathrm{THz}$ emission waveforms of $\mathrm{CdTe}$ on $\mathrm{AuAg}$ with different thicknesses are shown in Figure $3 \mathrm{c}$, and the strongest $\mathrm{THz}$ emission signal was also obtained from CdTe10@AuAg, which was $\sim 5$ and $\sim 3$ times stronger than that of the $\sim 5$ and $\sim 15 \mathrm{~nm}$ samples, respectively. It was interesting to find that the $\mathrm{THz}$ emission intensity is related to the Raman intensity from CdTe.

Since both the quantity of evaporated CdTe and the surface plasmon enhancement contributed to the Raman intensity [25,27], Raman mapping images of $\sim 5, \sim 10$, and $\sim 15 \mathrm{~nm}$ thick CdTe on the AuAg alloy films were collected and displayed in Figure 4 for further analysis, upon which 122 and $164 \mathrm{~cm}^{-1}$ were selected as the center wavenumbers. We observed that CdTe of $\sim 10 \mathrm{~nm}$ thickness was more evenly coated on the substrate and the average strength was stronger. At the beginning of the deposition process, randomly connected CdTe networks formed because of disordered nanometer-sized CdTe clusters [21,28], as shown in Figure 2a. With the coalescence and filling of CdTe, more 
homogeneous films were formed, showing an increased peak intensity of 122 and $164 \mathrm{~cm}^{-1}$ (Figure 3b). However, with the thickness further increased to $\sim 15 \mathrm{~nm}$ in Figure 3b, the peak intensity, conversely, decreased. Although thicker CdTe can effect better Raman signals, weakened SPP enhancement leads to totally decreased Raman intensity. Thus, it can be concluded that SPPs enhancement plays a key role in Raman scattering, where SPPs can generate the intense electric field to improve the optical signal of nearby materials [27]. Furthermore, SPPs are equally important for the enhancement of THz radiation; a similar variation trend can be observed in THz spectroscopy (Figure 3c). Thus, both the plasmonic enhancement and quality of thin CdTe film affect $\mathrm{THz}$ radiation capacity.

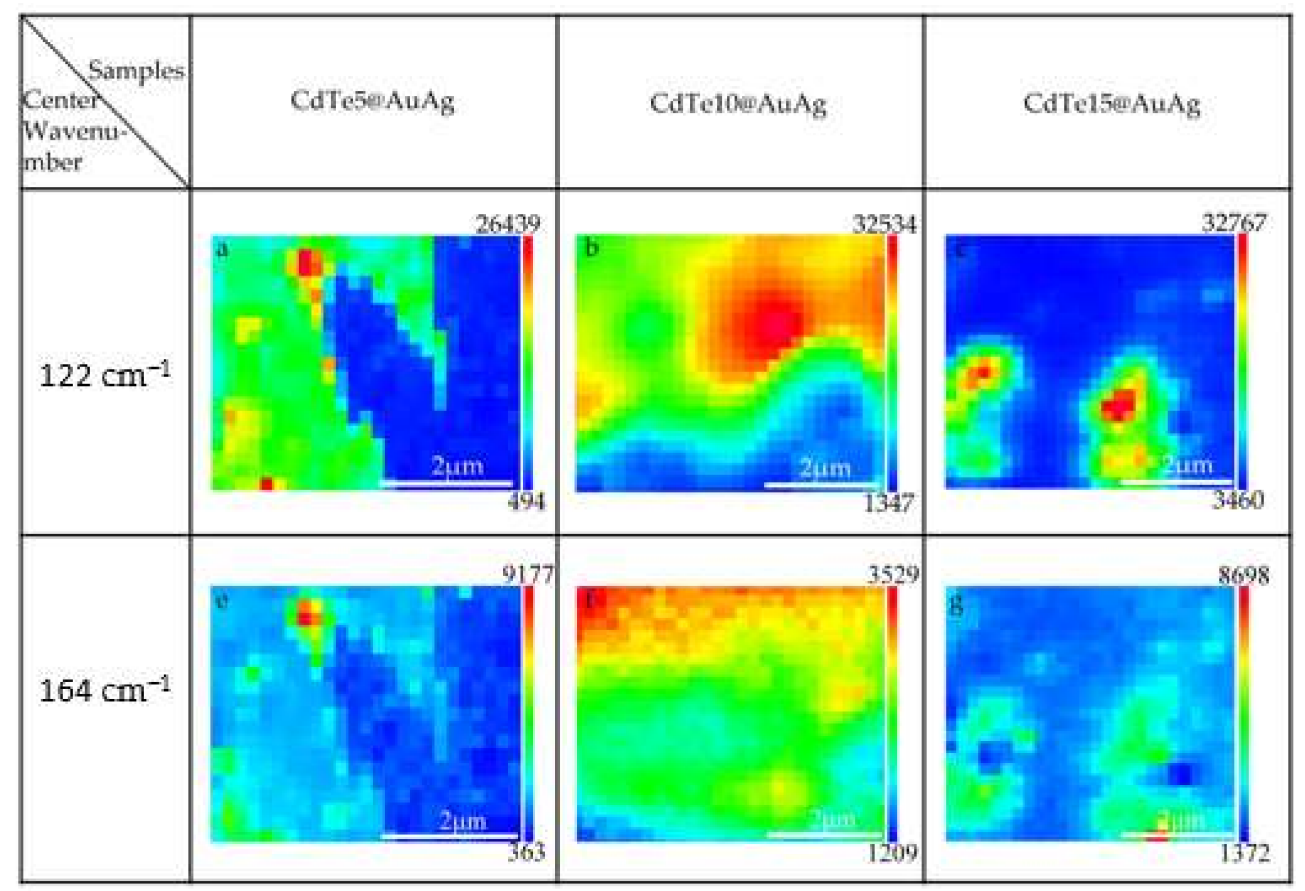

Figure 4. Raman mapping images of CdTe5@AuAg, CdTe10@AuAg, and CdTe15@AuAg films with characteristic peaks at $122 \mathrm{~cm}^{-1}$ and $164 \mathrm{~cm}^{-1}$.

The polarity, amplitude, and phase information of the generated $\mathrm{THz}$ wave reflected the linear and nonlinear optical properties, which can be used to analyze the mechanism of $\mathrm{THz}$ radiation and enhancement. The thin CdTe film with a thickness of $\sim 10 \mathrm{~nm}$ on the AuAg alloy was used for further $\mathrm{THz}$ wave measurement. When $780 \mathrm{~nm}$ laser pulses [27] illuminate the CdTe10@AuAg surface at an oblique incidence at $45^{\circ}$, ultrafast photocurrents are generated. These photocurrents consist of nonlinear currents $\left(J_{n l}\right)$, drift currents $\left(J_{d r i}\right)$ and diffusion currents $\left(J_{d i f}\right)$, which can be distinguished via THz emission signal. The surface depletion field $(E)$ is dominant to generate $J_{d r i}$, which can be described as $J_{d r i}=N e \mu E \propto P_{\text {photon, }}$, where $N$ is the density of the photo-generated carrier, $e$ is the electron charge, and $\mu$ is the mobility. The $E$ is irrelevant to pump power $\left(P_{\text {photon }}\right)$, while the density of the photogenerated carrier $N$ is proportional to $P_{\text {photon }}$. In addition, $J_{\text {dif }}$ is caused by the different mobility of the electrons and holes (the photo-Dember effect), and the currents can be described as $J_{d i f}=N e \mu E_{d} \propto P_{\text {photon, }}^{2}$ where $E_{d}$ is the field generated by the diffusion currents. According to the formula, the diffusion currents are proportional to the pump power squared, which is nonlinear [24,29]. Referring to our experimental results, shown in Figure 5a, the peak-valley value of the $\mathrm{THz}$ signal exhibited a linear relation with increasing the pump power. We can infer that it was the surface depletion field, not the diffusion currents, that made the main contribution to the photocurrent. 


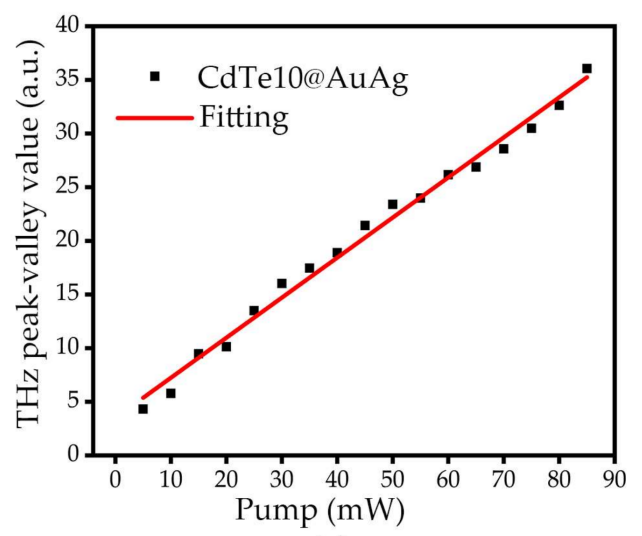

(a)

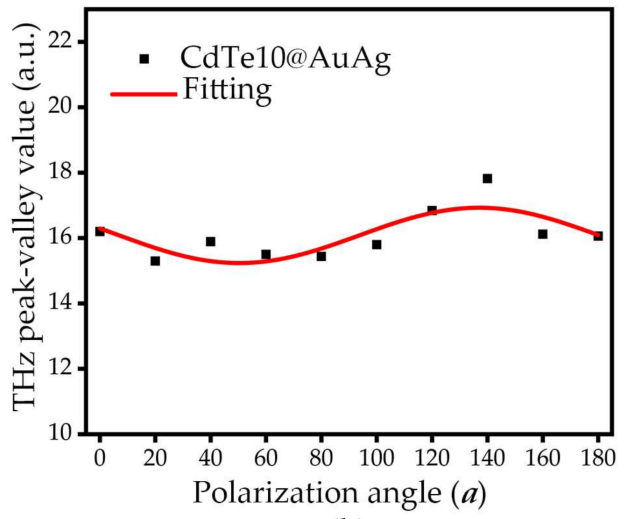

(b)

Figure 5. (a) THz peak-valley value from CdTe10@AuAg with respect to pump fluence; (b) tuning the pump polarization, the $\mathrm{THz}$ emission signal from peak to valley value in time-domain (the pump fluence used was $30 \mathrm{~mW}$ ).

Since the photo-generated nonlinear currents were more easily influenced by the polarization states of the ultrafast excitation pump beam, the variation of $\mathrm{THz}$ intensity with the polarization of the pump was used to analyze the contribution of the nonlinear effect to $\mathrm{THz}$ radiation [10]. The corresponding experimental results are shown in Figure 5b. A trigonometric relationship can be observed between the photocurrent and the polarization state of the laser pump. The results from the experiment reflect the non-linear property of $\mathrm{CdTe}$. According to the contribution of $\mathrm{THz}$ amplitude from the transient photocurrent and the current of nonlinear effect, as $E_{T H z}=C_{\text {current }}+C_{E F I O R} \cos 2 a$ [14], we can simply calculate that the contribution to $\mathrm{THz}$ emission of transient photocurrent was about 18 times larger than that of the nonlinear effect. In this paper, when SPPs were excited to enhance $\mathrm{THz}$ emission, CdTe preserved its nature.

\section{Conclusions}

We reported a THz emission enhancement of CdTe on pure metal and metal alloy thin films. The excited surface plasmon on $\mathrm{Au}$ and $\mathrm{AuAg}$ films was seen to particularly enhance THz emission by increasing the optical carrier density of CdTe. By combing with Raman scattering, we found that the thickness of CdTe exerted a slight effect on terahertz radiation and SPP enhancement mainly accounted for $\mathrm{THz}$ radiation enhancement. Our work suggests that it is possible to obtain stronger $\mathrm{THz}$ emissions from thin semiconductor films by providing a plasmonic basement and that the enhancement of the $\mathrm{THz}$ wave can be tuned by the components and surface morphology of metallic substrates.

Author Contributions: Conceptualization, methodology, preparation, H.K. and L.Z.; software, investigation, formal analysis, data curation, writing-original draft, H.K.; resources, L.Z. and M.L.; validation, supervision, project administration, funding acquisition, L.Z.; writing-review and editing, H.K., L.H., M.L., L.Z. and H.Z. All authors have read and agreed to the published version of the manuscript.

Funding: This study was supported by National Natural Science Foundation of China (No.61875126, 11727812).

Institutional Review Board Statement: Not applicable.

Informed Consent Statement: Not applicable.

Data Availability Statement: All data concerning this study are contained in the present manuscript or in previous articles, whose references have been provided.

Acknowledgments: The authors are very thankful to Jingquan Zhang from Sichuan University for supplying the CdTe films.

Conflicts of Interest: The authors declare no conflict of interest. 


\section{References}

1. Wallance, P.V.; MacPherson, E.; Zertler, J.A.; Reid, C. Three-dimensional Imaging of Optically Opaque Materials Using Nonionizing Terahertz Radiation. J. Opt. Soc. Am. A 2008, 25, 3120-3133. [CrossRef]

2. Shen, Y.C.; Taday, P.F. Development and Application of Terahertz Pulsed Imaging for Nondestructive Inspection of Pharmaceutical Tablet. IEEE J. Sel. Top. Quant. 2008, 14, 407-415. [CrossRef]

3. Shen, Y.C.; Lo, T.; Taday, P.F.; Cole, B.E.; Tribe, W.R.; Kemp, M.C. Detection and identification of explosives using terahertz pulsed spectroscopic imaging. Appl. Phys. Lett. 2004, 86, 24. [CrossRef]

4. Liu, J.L.; Dai, J.M.; Chin, S.L.; Zhang, X.C. Broadband terahertz wave remote sensing using coherent manipulation of fluorescence from asymmetrically ionized gases. Nat. Photonics 2010, 4, 627-631. [CrossRef]

5. Augustin, S.; Jung, P.; Hübers, H.W. Terahertz Dynamic Aperture Imaging at Standoff Distances Using a Compressed Sensing Protocol. IEEE Trans. Terahertz Sci. Technol. 2019, 9, 364-372. [CrossRef]

6. Nagatsuma, T.; Horiguchi, S.; Minamikata, Y.; Yoshimizu, Y.; Hisatake, S.; Kuwano, S.; Yoshimoto, N.; Terada, J.; Takahashi, H. Terahertz Wireless Communications based on Photonics Technologies. Opt. Express 2013, 21, 23736-23747. [CrossRef] [PubMed]

7. Huang, Y.Y.; Zhu, L.P.; Zhao, Q.Y.; Guo, Y.H.; Bai, J.T.; Xu, X.L. Surface Optical Rectification from Layered MoS 2 Crystal by THz Time-Domain Surface Emission Spectroscopy. ACS. Appl. Mater. Interfaces 2017, 9, 4956-4965. [CrossRef] [PubMed]

8. Arlauskas, A.; Treu, J.; Saller, K.; Beleckaitè, I.; Koblmüller, G.; Krotkus, A. Strong Terahertz Emission and Its Origin from Catalyst-Free InAs Nanowire Arrays. Nano Lett. 2014, 14, 1508-1514. [CrossRef]

9. Zhu, L.; Huang, Z.; Yao, Z.; Quan, B.; Li, J.; Gu, C.; Xu, X.; Ren, Z. Enhanced Polarization-Sensitive Terahertz Emission from Vertically Grown Graphene by a Dynamical Photon Drag Effect. Nanoscale 2017, 9, 10301-10311. [CrossRef] [PubMed]

10. Kumar, N.; Abdi, F.F.; Trzesniewski, B.; Smith, W.A.; Planken, P.C.M.; Adam, A.J.L. Investigation of Terahertz Emission from $\mathrm{BiVO}_{4} / \mathrm{Au}$ Thin Film Interface. J. Infrared Millim. Terahertz Waves 2015, 36, 1033-1042. [CrossRef]

11. Norku, R.; Nevinskas, I.; Krotkus, A. Spectral Dependencies of Terahertz Emission from Femtosecond Laser Excited Surfaces of Germanium crystals. J. Appl. Phys. 2019, 126, 22.

12. Ramakrishnan, G.; Kumar, N.; Ramanandan, G.K.P.; Adam, J.L.A.; Hendrikx, W.A.R.; Planken, C.M.P. Plasmon-Enhanced Terahertz Emission from a Semiconductor/metal Interface. Appl. Phys. Lett. 2014, 104, 7. [CrossRef]

13. Bahk, Y.M.; Ramakrishnan, G.; Choi, J.; Song, H.; Choi, G.; Kim, Y.H.; Ahn, K.J.; Kim, D.S.; Planken, P.C. Plasmon Enhanced Terahertz Emission from Single Layer Graphene. J. Am. Chem. Soc. 2014, 8, 9089-9096. [CrossRef]

14. Huang, L.Y.; Zhang, L.; Zhou, J.Z.; Li, M.; Li, C.; Li, C.; Zhang, J.; Wang, S.; Zeng, H. Surface Plasmon Enhanced THz Emission with Nanoporous gold Supported CdTe. Opt. Express 2021, 29, 19853-19861. [CrossRef] [PubMed]

15. Hakeem, A.M.A.; Ali, H.M.; El-Raheem, M.M.A.; Hasaneen, M.F. Study the Effect of Type of Substrates on the Microstructure and Optical Properties of CdTe Thin Films. Optik 2021, 225, 165390. [CrossRef]

16. Arce-Plaza, A.; Sānchez-Rodrìguez, F.J.; Courel, M.; Pérez-Cuapio, R.; Alvarado, J.A.; Roque, J.; Ortega-López, M. CdTe Colloidalgel: Synthesis and Thin Films Deposition Applied to Solar Cells. Mater. Sci. Semicond. Process. 2021, 131, 105871. [CrossRef]

17. Sedzicki, P.; Skowronski, L.; Szczesny, R.; Becker, H.; Rogalla, D.; Pawlak, M.; Derkowska-Zielinska, B.; Lukasz, S. Influence of Phosphorus ion Implantation on the Optical Properties of CdTe Bulk Crystal. J. Alloys Compd. 2020, 55, 7715-7730. [CrossRef]

18. Rahman, M.F.; Hossain, J.; Kuddus, A.; Tabassum, S.; Rubel, M.H.K.; Moriya, Y.; Shirai, H.; Ismail, A.B. A Novel CdTe Ink-assisted Direct Synthesis of CdTe Thin Films for the Solution-Processed CdTe Solar Cells. J. Mater. Sci. 2020, 55, 7715-7730. [CrossRef]

19. Dharmadasa, I.M.; Echendu, O.K.; Fauzi, F.; Abdul-Manaf, N.A.; Olusola, O.I.; Salim, H.I.; Madugu, M.L.; Ojo, A.A. Improvement of Composition of CdTe Thin Films during Heat Treatment in the Presence of $\mathrm{CdCl}_{2}$. J. Mater. Sci. Mater. Electron. 2017, 28, 2343-2352. [CrossRef]

20. Pine, A.S.; Dresselhaus, G. Raman Spectra and Lattice Dynamics of Tellurium. Phys. Rev. B 1971, 4, 356-371. [CrossRef]

21. Himanshu; Patel, S.L.; Thakur, A.; Kannan, M.D.; Dhaka, M.S. Analysis of Different Annealing Conditions on Physical Properties of Bi Doped CdTe Thin Films for Potential Absorber Layer in Solar Cells. Sol. Energy 2020, 199, 772-781. [CrossRef]

22. Liu, X.F.; Yu, H.Y.; Ji, Q.Q.; Ge, S.F.; Qiu, J.; Liu, Z.F.; Zhang, Y.F.; Sun, D. An Ultrafast Terahertz Probe of the Transient Evolution of the Charged and Neural Phase of Photo-excited Electron-hole gas in a Monolayer Semiconductor. 2D Mater. 2016, 3, 014001. [CrossRef]

23. Ramakrishnan, G.; Ramanandan, G.K.P.; Adam, J.L.A.; Xu, M.; Kumar, N.; Hendrikx, R.W.A.; Planken, P.C.M. Enhanced Terahertz Emission by Coherent Optical Absorption in Ultrathin Semiconductor Films on Metals. Opt. Express 2013, 21, 16784-16798. [CrossRef]

24. Fang, Z.; Wang, H.; Wu, X.; Shan, S.; Wang, C.; Zhao, H.; Xia, C.; Nie, T.; Miao, J.; Zhang, C.; et al. Nonlinear Terahertz Emission in the Three-Dimensional Topological Insulator Bi2Te3 by Terahertz Emission Spectroscopy. Appl. Phys. Lett. 2019, 115, 19. [CrossRef]

25. Proenca, M.; Borges, J.; Rodrigues, M.S.; Meira, D.; Sampaio, P.; Dias, J.P.; Pedrosa, P.; Martin, N.; Bundaleski, N.; Teodoro, O.M.; et al. Nanocomposite Thin Films based on Au-Ag Nanoparticles Embedded in a CuO Matrix for Localized Surface Plasmon Resonance Sensing. Appl. Surf. Sci. 2019, 484, 152-168. [CrossRef]

26. Li, A.B.; Peng, Z.; Zheng, J.X.; Pan, F. Optimizing CdTe-metal Interfaces for High Performance Solar Cells. J. Mater. Chem. A 2017, 5, 7118-7124. [CrossRef]

27. Daza, L.G.; Martìn-Tovar, E.A.; Escobedo-Vera, R.; Castro-Rodríguez, R.; Iribarren, A. Improved Optical Absorption by Local Surface Plasmon Resonance of Silber Nanoparticle in Nanocolumnar CdTe Thin Films. Indian J. Phys. 2022, 96, 257-265. [CrossRef] 
28. Major, J.D. Grain Boundaries in CdTe Thin Film Solar Cells: A Review. Semicond. Sci. Technol. 2016, 31, 9. [CrossRef]

29. Yao, Z.H.; Huang, Y.Y.; Zhu, L.P.; Obraztsov, P.A.; Du, W.Y.; Zhang, L.H.; Xu, X.L. Interfacial THz Generation from Graphene/Si Mixed-Dimensional van der Waals Heterostructure. Nanoscale 2019, 11, 16614-16620. [CrossRef] [PubMed] 